\title{
A theoretical study of how C2-substitution affects alkaline stability in imidazolium-based anion exchange membranes
}

\author{
Ryo Tsuchitani ${ }^{\mathrm{a}}$, Hiroshi Nakanishi ${ }^{\mathrm{a}}$, Hideyuki Shishitani ${ }^{\mathrm{b}}$, \\ Susumu Yamaguchi ${ }^{\mathrm{b}}$, Hirohisa Tanaka ${ }^{\mathrm{b}}$, Hideaki Kasai ${ }^{\mathrm{a}, \mathrm{c}, \mathrm{d}, *}$ \\ ${ }^{a}$ Department of Applied Physics, Osaka University, 2-1 Yamadaoka, Suita, Osaka \\ 565-0871, Japan \\ ${ }^{b} R$ \& D Division, Daihatsu Motor Co., Ltd., 3000 Yamanoue, Ryuo, Gamo, Shiga \\ 520-2593, Japan \\ ${ }^{c}$ Center for Atomic and Molecular Technologies, Osaka University, 2-1 Yamadaoka, Suita, \\ Osaka 565-0871, Japan \\ ${ }^{d}$ Center for Continuing Professional Development, Osaka University, 2-1 Yamadaoka, \\ Suita, Osaka 565-0871, Japan
}

\begin{abstract}
We use density functional theory and investigate the effect on C2-substitution in imidazolium-based anion exchange membranes (AEMs). Here, a C2-unsubstituted 1-ethyl-3-(4-isopropylbenzyl) imidazolium cation (EIIM), a 1-ethyl-2-methyl3-(4-isopropylbenzyl) imidazolium cation (EMIIM), a 1-ethyl-2-isopropyl-3-(4isopropylbenzyl) imidazolium cation (EIIIM) and a 1-ethyl-2-phenyl-3-(4-isopropylbenzyl) imidazolium cation (EPhIIm) are prepared. In our previous paper, we suggested that C2-unsubstituted EIIM was quite sensitive to a deprotonation reaction because it was thermodynamically downhill. In the present work, we calculate the alkaline stability of the $\mathrm{C} 2$-substituted imidazolium cations and clarify which part is most affected by the C2-substitution. First, in order to investigate the effect on the steric hindrance by each substituent, potential energy curves are described. The effect appears explicitly in the C2-substituted imidazolium cations. Second, the minimum energy paths along the ring-opening and deprotonation reactions are calculated. Finally, we conclude that the alkaline stability is in the order of EMIIM $>$ EIIIM $>$ EPhIIM $>$ EIIM. Furthermore, the order of
\end{abstract}

\footnotetext{
* Corresponding author

Email address: kasai@dyn.ap.eng.osaka-u.ac.jp (Hideaki Kasai)
}

Preprint submitted to Journal of ${ }^{A} T_{E} X$ Templates

January 26, 2015

(C) 2015. This manuscript version is made available under the Elsevier user license http://www.elsevier.com/open-access/userlicense/1.0/ 
the alkaline stability is consistent with that of the experiment performed by Lin et al.

Keywords: Density functional theory, C2-substitution, Imidazole, Steric hindrance, Alkaline stability

\section{Introduction}

Proton exchange membrane fuel cells (PEMFCs) have been focused as one of the most promising environment-friendly energy sources. They have the great potential to apply to automobiles and domestic electric power supply due to

5 their high power density, high energy conversion efficiencies and low operation temperature. Nafion perfluorosulfonic acid membranes which are most popular as proton exchange membranes show high alkaline stability, high ionic conductivity and long durability.[1] However, PEMFCs need noble metal catalysts such as platinum for acid environment, resulting in high cost and the cost prevents PEMFCs from being commercialized. Therefore, there is a high expectation for anion exchange membrane fuel cells (AEMFCs) where $\mathrm{OH}^{-}$or other anions pass through anion exchange membranes (AEMs) instead of protons.

The detail mechanism of AEMFCs with typical hydrogen fuel is the following.

$$
\begin{array}{r}
\text { Anode }: \mathrm{H}_{2}+2 \mathrm{OH}^{-} \rightarrow 2 \mathrm{H}_{2} \mathrm{O}+2 \mathrm{e}^{-} \\
\text {Cathode }: 1 / 2 \mathrm{O}_{2}+\mathrm{H}_{2} \mathrm{O}+2 \mathrm{e}^{-} \rightarrow 2 \mathrm{OH}^{-} \\
\text {Overall }: \mathrm{H}_{2}+1 / 2 \mathrm{O}_{2} \rightarrow \mathrm{H}_{2} \mathrm{O}
\end{array}
$$

AEMFCs can use non-precious metal such as nickel[2] and cobalt[3] to catalysts for alkaline environment and overcome the tender point of PEMFCs in the aspect of the cost. However, AEMFCs also have some problems. The most serious issue is alkaline stability of AEMs and they are very sensitive to $\mathrm{OH}^{-}$ attack. In particular, $\mathrm{OH}^{-}$anions lead to faster degradation of AEMs in the absence of water at elevated temperature. Hence, the lifetime of the AEMs 
gets very short and AEMFCs are still inadequate to spread widely to societies. There are many kinds of AEMs such as quaternary ammonium (QA)[4], guanidinium[5], pyridinium[6] and imidazolium[7] cations. Although QA cations are most common among anion exchange groups, their alkaline stability is poor 25 due to some degradation pathways ( $\mathrm{S}_{\mathrm{N}} 2$ and Hoffmann elimination).[8, 9] Thus, various cations based on AEMs have been investigated both experimentally and theoretically.

Recently, it has been reported that imidazolium cations raise the durability to $\mathrm{OH}^{-}$attack due to the presence of the $\pi$-conjugated imidazole ring compared with quaternary ammonium cations.[10] The main degradation reaction in imidazolium-based AEMs is called ring-opening reaction, which is a kind of nucleophilic substitution reaction. $\mathrm{OH}^{-}$anions attack the $\mathrm{C}$ between the two $\mathrm{N}$ atoms of the imidazole ring $(\alpha-\mathrm{C})$ under alkaline condition and then the imidazole ring is broken. AEMs degraded by $\mathrm{OH}^{-}$attack lose the ability in ion conductivity. Lin et al. revealed that the alkaline stability of $\mathrm{C} 2$-substituted imidazolium cations substituted by methyl, isopropyl and phenyl groups is significantly enhanced compared with C2-unsubstituted imidazolium cations due to the effect on the steric hindrance by their substituents.[11] Steric hindrance plays a critical role in physically protecting imidazolium cations against $\mathrm{OH}^{-}$ 40 attack. Hence, C2-substitution is an important factor to enhance the alkaline stability of AEMs. In addition to the alkaline stability, the ionic conductivity also has been investigated.[12, 13] However, the condition of ideal substituents which satisfies both of them is still unclear and the present situation cannot help depending on trial and error.

In our previous paper, the alkaline stability of a $\mathrm{C} 2$-unsubstituted imidazolium cation was numerically investigated.[14] The results showed that although the $\mathrm{C} 2$-substituted imidazolium cation has the resistance to the ringopening reaction, it was quite sensitive to a deprotonation reaction due to thermodynamically downhill. Hence, we concluded that the deprotonation reaction was an initial degradation reaction before the ring-opening reaction and it was much more dominant rather than the ring-opening reaction. 
Based on density functional theory (DET), in the present analysis, we theoretically investigate the effect on $\mathrm{C} 2$-substitution and the alkaline stability in $\mathrm{C} 2$-substituted imidazolium cations. In order to compare our results with that of the experiment performed by Lin et al., One C2-unsubstituted and three C2substituted imidazolium cations substituted by methyl, isopropyl and phenyl groups are prepared and their alkaline stability is compared to each other. In particular, we focus on how the energy barriers for the two degradation pathways of the ring-opening and deprotonation reactions change by each substituent.

\section{Computational Method}

We use Gaussian 09 (G09)[15] to optimize structures and calculate the energies for the initial state (IS), transition state (TS) and final state (FS) structures. For a model reaction $\mathrm{A}+\mathrm{B} \rightarrow \mathrm{C}$, we optimize the geometries of species $\mathrm{A}, \mathrm{B}$, and $\mathrm{C}$ in a dielectric medium of $\varepsilon=80$ (corresponding to water). During the calculations, we use the DFT B3LYP method, 6-311++G(2d,p) basis set, and polarizable continuum solvation model (PCM) implemented in G09. In addition, we use the natural bond orbital population scheme for molecular orbital analysis and atomic charge calculations. A temperature of $80{ }^{\circ} \mathrm{C}$ is used in all calculations because the operating temperature of AEMFCs is around $80^{\circ} \mathrm{C}$ and imidazolium-based AEMs are also degraded at elevated temperature. We then compute the force constants based on the optimized structure, resulting in the vibrational frequencies and Gibbs free energies $G$. $G$ is defined as $G=H-T S$, where $H, T$ and $S$ are enthalpy, temperature and entropy, respectively.

$G$ is calculated by adding the following terms to $E$ : cavity formation energy, van der Waals interactions, translational free energy, quantum mechanical rotational and vibrational free energies, and zero point energy, all as reported by G09 software. The self-consistent field energy $E$ reported by G09 includes the electronic energy of the solute and the interactions with the dielectric medium. The energy change $\Delta E$ and $\Delta G^{0}$ are defined as, $\Delta E=E(\mathrm{C})-(E(\mathrm{~A})+E(\mathrm{~B}))$ so and $\Delta G^{0}=G^{0}(\mathrm{C})-\left(G^{0}(\mathrm{~A})+G^{0}(\mathrm{~B})\right)$. Additionally, the energy barrier $\Delta E^{\neq}$ 
and $\Delta G^{\neq, 0}$ are defined as, $\Delta E^{\neq}=E\left([\mathrm{AB}]^{\neq}\right)-(E(\mathrm{~A})+E(\mathrm{~B}))$ and $\Delta G^{\neq, 0}=$ $G^{0}\left([\mathrm{AB}]^{\neq}\right)-\left(G^{0}(\mathrm{~A})+G^{0}(\mathrm{~B})\right) \cdot[\mathrm{AB}]^{\neq}$is the exact TS (the saddle point on the potential energy surface) and it is obtained from this approximate point by using the Berny optimization algorithm as implemented in G09.

\section{Results and Discussion}

Referring the paper of Lin et al., we use the same C2-substituents as their experiment and evaluate the alkaline stability. First, four geometries are optimized as shown in Fig. 1. Figure 1(A) is a C2-unsubstituted 1-ethyl-3-(4isopropylbenzyl) imidazolium cation (EIIM). Figures 1 (B), (C) and (D) are a 1-ethyl-2-methyl-3-(4-isopropylbenzyl) imidazolium cation (EMIIM), a 1-ethyl2-isopropyl-3-(4-isopropylbenzyl) imidazolium cation (EIIIM) and a 1-ethyl-2phenyl-3-(4-isopropylbenzyl) imidazolium cation (EPhIIM), respectively. Second, we describe potential energy curves (PECs) and investigate the effect on the steric hindrance in the $\mathrm{C} 2$-substituted imidazolium cations. Finally, the minimum energy paths along the ring-opening and deprotonation reactions are calculated and it is found that the $\mathrm{C} 2$-substitution can significantly enhance the alkaline stability of the deprotonation reaction.

In order to investigate the effect on the steric hindrance quantitatively, PECs of each imidazolium cation are shown in Fig. 2. Here, the reference $(\Delta E=0)$ is the sum of the energy of the separated reactants. If necessary, we describe the PECs by a method; we assume that an $\mathrm{OH}^{-}$anion approaches to the $\alpha$-C and then the ring-opening reaction occurs. Thus, we fix the distance between the $\alpha$ $\mathrm{C}$ and the $\mathrm{O}$ of the $\mathrm{OH}^{-}$anion $d$ and calculate the potential energy as a function of $d$, changing $d$ from 3.5 to $1.5 \AA$ with every $0.1 \AA$. Figure 2 indicates that in the case of EIIM, the approach of an $\mathrm{OH}^{-}$anion makes the potential energy continue to decrease monotonically due to the attractive interaction between the $\mathrm{H}$ bonding to the $\alpha$-C and the $\mathrm{O}$ of the $\mathrm{OH}^{-}$anion, which eventually results in the deprotonation reaction at $d=2.6 \AA$ as shown in Fig. 3. Hence, the alkaline stability of EIIM is by far the lowest. On the other hand, the effect on the steric 
hindrance appears obviously in EMIIM, EIIIM and EPhIIM due to the repulsive interaction between the $\mathrm{OH}^{-}$anion and every substituent. The highest points of EMIIM, EPhIIM and EIIIM are 0.21, 0.27 and $0.29 \mathrm{eV}$, respectively. The larger the size of the substituent is, the larger the effect on the steric hindrance also tends to become. This is because the isopropyl and phenyl groups consist of a larger number of atoms than the methyl group and the repulsive interaction between electrons becomes stronger. The $\pi$-electrons generated by the benzene ring in EPhIIM are weakly-bounded to the nucleus and it is easy to move freely. Thus, when an $\mathrm{OH}^{-}$anion approaches to the $\alpha$-C, the $\pi$-electrons intend to avoid the $\mathrm{OH}^{-}$anion and then the potential energy in EPhIIM gets a little smaller than that in EIIIM. Hence, EIIIM has the largest effect on the steric hindrance among the C2-substituted imidazolium cations. Consequently, the effect on the steric hindrance is in the order of EIIIM $>$ EPhIIM $>$ EMIIM $>$ EIIM. From the analyses of the PECs, EIIM is exceedingly vulnerable to attack from the $\mathrm{OH}^{-}$anion and all of the following calculations are performed in only the C2-substituted imidazolium cations.

Using the $\mathrm{C} 2$-substituted imidazolium cations, we calculate the minimum energy paths along the ring-opening reaction. The reference $(\Delta G=0)$ is also the sum of the Gibbs free energy of the separated reactants in common with $\Delta E=0$. Figure 4 demonstrates that all of the energy paths are very similar. 30 The energy barriers in EMIIM, EIIIM and EPhIIM are 1.53, 1.50 and $1.48 \mathrm{eV}$, respectively as shown in Table 1 . They are almost comparable to each other and the differences can be negligible. This result shows that the $\mathrm{C} 2$-substitution plays the role of the steric hindrance and hinders the $\mathrm{OH}^{-}$attack only when an $\mathrm{OH}^{-}$anion approaches to the $\alpha$-C of an imidazole ring. Once the ring-opening reaction begins to occur, the differences between the substituents do not depend on the energy barriers. Although the differences between the energy barriers are quite little, the alkaline stability is in the order of EMIIM > EIIIM > EPhIIM. Furthermore, the step $(\mathrm{E})$ to $(\mathrm{G})$ corresponds to the situation of the approach of an $\mathrm{OH}^{-}$anion as shown in Fig. 2. The highest steps (F) of EMIIM, EPhIIM and EIIIM are $0.21,0.25$ and $0.31 \mathrm{eV}$, respectively in terms of $\Delta E$ and those are 
closely consistent with the highest points of Fig. 2. Nucleophilic substitution reaction is affected by the lowest unoccupied molecular orbital (LUMO) energy of a compound and the lower the LUMO energy is, the easer the nucleophilic attack becomes.[16] In the case of imidazolium cations, the lower LUMO energy of the compound makes $\mathrm{OH}^{-}$attack easer and the durability of the ring-opening reaction decreases. Figure 5 shows that the LUMO energy of EMIIM, EIIIM and EPhIIM are $-1.029,-1.080$ and $-1.439 \mathrm{eV}$, respectively. EMIIM has the highest LUMO energy because the hyper-conjugation between the $\pi$-conjugated imidazole ring and the $\sigma$ bond of the methyl group increases the electron density at the imidazolium cation.[17] Hence, EMIIM can make $\mathrm{OH}^{-}$attack most difficult. On the other hand, EPhIIM has the lowest LUMO energy because the electronwithdrawing effect by the phenyl group decreases the electron density at the imidazolium cation.[18] The order of the LUMO energy in the C2-substituted imidazolium cations is completely consistent with that of the energy barriers of the ring-opening reaction.

As well as the ring-opening reaction, we also calculate the minimum energy paths along the deprotonation reaction. Figure 6 demonstrates that the differences between the substituents depend on the energy barriers sensitively in contrast to the ring-opening reaction. Table 2 shows that the energy barriers in EMIIM, EIIIM and EPhIIM are 3.83, 3.47 and $2.15 \mathrm{eV}$, respectively and all of them are very high. The energy changes of EMIIM and EIIIM are 0.43 and $0.50 \mathrm{eV}$, respectively, indicating that the ISs are most stable. Although the energy change of EPhIIM is $-0.05 \mathrm{eV}$, the reaction is highly reversible. Hence, C2-substitution can hinder the deprotonation reaction and enhance the alkaline stability dramatically. The deprotonation reaction is triggered by $\mathrm{OH}^{-}$attack to the $\mathrm{C}$ bonding to the $\alpha-\mathrm{C}(\beta-\mathrm{C})$. Table 3 shows the electron density distribution at the $\alpha$-C and $\beta$-C. The electron density distribution at the $\beta$ - $\mathrm{C}$ in EMIIM, EIIIM and EPhIIM is $-0.634,-0.278$ and -0.141 , respectively and there are considerable differences. In particular, the electron density distribution at the $\beta$-C of EMIIM is much higher than the other cations because the $\beta$-C has three $\mathrm{H}$ atoms and it can attract most electrons from the $\mathrm{H}$ atoms due to the dif- 


\begin{tabular}{c|cccc}
\hline & $\Delta E(\mathrm{eV})$ & $\Delta G^{0}(\mathrm{eV})$ & $\Delta E^{\neq}(\mathrm{eV})$ & $\Delta G^{\neq, 0}(\mathrm{eV})$ \\
\hline EMIIM & -1.05 & -0.49 & 1.10 & 1.53 \\
EIIIM & -1.14 & -0.67 & 0.98 & 1.50 \\
EPhIIM & -1.06 & -0.49 & 0.93 & 1.48 \\
\hline
\end{tabular}

Table 1: Calculated energy changes and energy barriers in the ring-opening reaction. There are little differences between the energy barriers. Energy is given by $\mathrm{eV}$.

ference between the electronegativities of $\mathrm{C}$ and $\mathrm{H}$, which results in the highest energy barrier. The $\beta$-C of EIIIM has one $\mathrm{H}$ atom and the electron density distribution is lower than that of EMIIM. Thus, the repulsive interaction between electrons gets weaker and then the energy barrier is lower than that of EMIIM. However, the $\beta$-C of EPhIIM has no $\mathrm{H}$ atoms and cannot attract electrons from $\mathrm{H}$ atoms. As the result, the electron density distribution at the $\beta$-C decreases and the energy barrier is lowest. Hence, the alkaline stability of the deprotonation reaction is in the order of EMIIM $>$ EIIIM $>$ EPhIIM. Incidentally, Table 3 also shows that the electron density distribution at the $\alpha$-C is almost equivalent. This is because the number and the element of the atoms bonding to the $\alpha-\mathrm{C}$ are common regardless of the differences between the substituents. To be specific, all of the $\alpha$-C have one $\mathrm{C}$ and two $\mathrm{N}$ atoms. Thus, the energy barriers of the ring-opening reaction are almost independent of the differences between the substituents as shown in Table 1. Consequently, C2-substitution makes a most effective influence on the electron density distribution at the $\beta$ C. Therefore, the C2-substitution exerts a most important effect to hinder the deprotonation reaction and eventually the alkaline stability can be enhanced considerably compared with that of C2-unsubstitution.

\section{Conclusion}

Based on DFT method, it is numerically investigated how C2-substitution affects the alkaline stability in imidazolium cations. First of all, we prepare four imidazolium cations and PECs are described. They indicate that the effect 


\begin{tabular}{c|cccc}
\hline & $\Delta E(\mathrm{eV})$ & $\Delta G^{0}(\mathrm{eV})$ & $\Delta E^{\neq}(\mathrm{eV})$ & $\Delta G^{\neq, 0}(\mathrm{eV})$ \\
\hline EMIIM & 0.08 & 0.43 & 3.46 & 3.83 \\
EIIIM & -0.33 & 0.50 & 3.22 & 3.47 \\
EPhIIM & -0.46 & -0.05 & 1.56 & 2.15 \\
\hline
\end{tabular}

Table 2: Calculated energy changes and energy barriers in the deprotonation reaction. In contrast to the ring-opening reaction, the differences between the substituents depend on the energy barriers. Energy is given by eV.

\begin{tabular}{c|cc}
\hline electron density distribution & $\alpha$-C & $\beta$-C \\
\hline EMIIM & 0.506 & -0.634 \\
EIIIM & 0.516 & -0.278 \\
EPhIIM & 0.488 & -0.141 \\
\hline
\end{tabular}

Table 3: Electron density distribution at the $\alpha$-C and $\beta$-C in EMIIM, EIIIM and EPhIIM.

on the steric hindrance appears explicitly in the $\mathrm{C} 2$-substituted imidazolium cations as several experimental groups point out. Next, the minimum energy paths along the ring-opening and deprotonation reactions are calculated. The energy barriers of the ring-opening reaction are almost comparable to each other. Hence, they are independent of the differences between the substituents. However, the energy barriers of the deprotonation reaction depend on the differences between the substituents because the differences seriously affect the electron density distribution at the $\beta$-C.

Considering the comprehensive evaluation of the PECs and the energy paths along both the ring-opening and deprotonation reactions, the alkaline stability is in the order of EMIIM $>$ EIIIM $>$ EPhIIM $>$ EIIM because EMIIM can hinder the deprotonation reaction most effectively among the $\mathrm{C} 2$-substituted imidazolium cations due to the highest electron density distribution at the $\beta$-C. The order is consistent with that of the experiment by Lin et al. We conclude that the most important effect of $\mathrm{C} 2$-substitution is to increase the electron density at the $\beta$ - $\mathrm{C}$ and hinder the deprotonation reaction. Therefore, the methyl 

imidazolium cations.

\section{Acknowledgements}

This work is supported in part by: Daihatsu Motor Corporation, MEXT Grant-in-Aid for Scientific Research on Innovative Areas Program (2203-22104008), and Scientific Research programs (A) (24246013) and (C) (22510107); JST ALCA Program Development of Novel Metal-Air Secondary Battery Based on Fast Oxide Ion Conductor Nano Thickness Film" and Strategic Japan-Croatia Research Cooperative Program on Materials Sciences Program " Theoretical Modeling and Simulations of the Structural, Electronic, and Dynamical Propthe calculations presented here were done using the computer facilities at the following institutes: ISSP Supercomputer Center (University of Tokyo); Cybermedia center (Osaka University); Yukawa Institute (Kyoto University); High Energy Accelerator Research Organization (KEK); and the National Institute ${ }_{225}$ for Fusion Science (NIFS).

\section{References}

[1] Michael A. Hickner, Hossein Ghassemi, Yu Seung Kim, Brian R. Einsla, James E. McGrath, Chem. Rev. 104 (2004) 4587-4612.

[2] Tomokazu Sakamoto, Koichiro Asazawa, Ulises Martinez, Barr Halevi, Toshiyuki Suzuki, Shigeo Arai, Daiju Matsumura, Yasuo Nishihata, Plamen Atanassov, Hirohisa Tanaka, Journal of Power Sources 234 (2013) 252-259.

[3] M. Mamlouk, S. M. Senthil Kumar, Pascal Gouerec, Keith Scott, Journal of Power Sources 196 (2011) 7594-7600.

[4] Junhua Wang, Zhuo Zhao, Feixiang Gong, Shenghai Li, Suobo Zhang, 235 Macromolecules 42 (2009) 8711-8717. 
[5] Lei Liu, Qing Li, Jingwen Dai, Hu Wang, Bangkun Jin, Ruke Bai, Journal of Membrane Science 453 (2014) 52-60.

[6] Sandip Maurya, Sung-Hee Shin, Ki-Won Sung, Seung-Hyeon Moon, Journal of Power Sources 255 (2014) 325-334.

[7] Wenpin Wang, Shubo Wang, Xiaofeng Xie, Yafei Iv, Vijay Ramani, International Journal of Hydrogen Energy 39 (2014) 14355-14361.

[8] Hai Long, Kwiseon Kim, Bryan S. Pivovar, J. Phys. Chem. C 116 (2012) 9419-9426.

[9] Shaji Chempath, James M. Boncella, Lawrence R. Pratt, Neil Henson, Bryan S. Pivovar, J. Phys. Chem. C 114 (2010) 11977-11983.

[10] Yuesheng Ye, Yossef A. Elabd, Macromolecules 44 (2011) 8494-8503.

[11] Bencai Lin, Huilong Dong, Youyong Li, Zhihong Si, Fenglou Gu, Feng Yan, Chem. Mater. 25 (2013) 1858-1867.

[12] K. Yoshimura, H. Koshikawa, T. Yamaki, H. Shishitani, K. Yamamoto, S. Yamaguchi, H. Tanaka, Y. Maekawa, J. Electrochem. Soc. 161 (2014) F889-F893.

[13] Christopher G. Arges, Javier Parrondo, Graham Johnson, Athrey Nadhan, Vijay Ramani, J. Mater. Chem. 22 (2012) 3733-3744.

[14] Ryo Tsuchitani, Hiroshi Nakanishi, Hideaki Kasai, e-J. Surf. Sci. Nanotech. 11 (2013) 138-141.

[15] M. J. Frisch, G. W. Trucks, H. B. Schlegel, G. E. Scuseria, M. A. Robb, J. R. Cheeseman, G. Scalmani, V. Barone, B. Mennucci, G. A. Petersson, H. Nakatsuji, M. Caricato, X. Li, H. P. Hratchian, A. F. Izmaylov, J. Bloino, G. Zheng, J. L. Sonnenberg, M. Hada, M. Ehara, K. Toyota, R. Fukuda, J. Hasegawa, M. Ishida, T. Nakajima, Y. Honda, O. Kitao, H. Nakai, T. Vreven, J. A. Montgomery, Jr., J. E. Peralta, F. Ogliaro, M. Bearpark, 
J. J. Heyd, E. Brothers, K. N. Kudin, V. N. Staroverov, R. Kobayashi, J. Normand, K. Raghavachari, A. Rendell, J. C. Burant, S. S. Iyengar, J. Tomasi, M. Cossi, N. Rega, J. M. Millam, M. Klene, J. E. Knox, J. B. Cross, V. Bakken, C. Adamo, J. Jaramillo, R. Gomperts, R. E. Stratmann, O. Yazyev, A. J. Austin, R. Cammi, C. Pomelli, J. W. Ochterski, R. L. Martin, K. Morokuma, V. G. Zakrzewski, G. A. Voth, P. Salvador, J. J. Dannenberg, S. Dapprich, A. D. Daniels, . Farkas, J. B. Foresman, J. V. Ortiz, J. Cioslowski, D. J. Fox, Gaussian 09, Revision D.01, Gaussian, Inc., Wallingford CT, 2009.

[16] Markus Pernpointner, A. Stephen K. Hashmi, J. Chem. Theory Comput. 5 (2009) 2717-2725.

[17] Exner O and Böhm S, J. Chem. Soc., Perkin Trans. 2 (1997) 1235-1240.

[18] Wenpin Wang, Shubo Wang, Xiaofeng Xie, Yafei Iv and Vijay Ramani, International Journal of Hydrogen Energy 39 (2014) 14355-14361. 


\section{FIGURES}
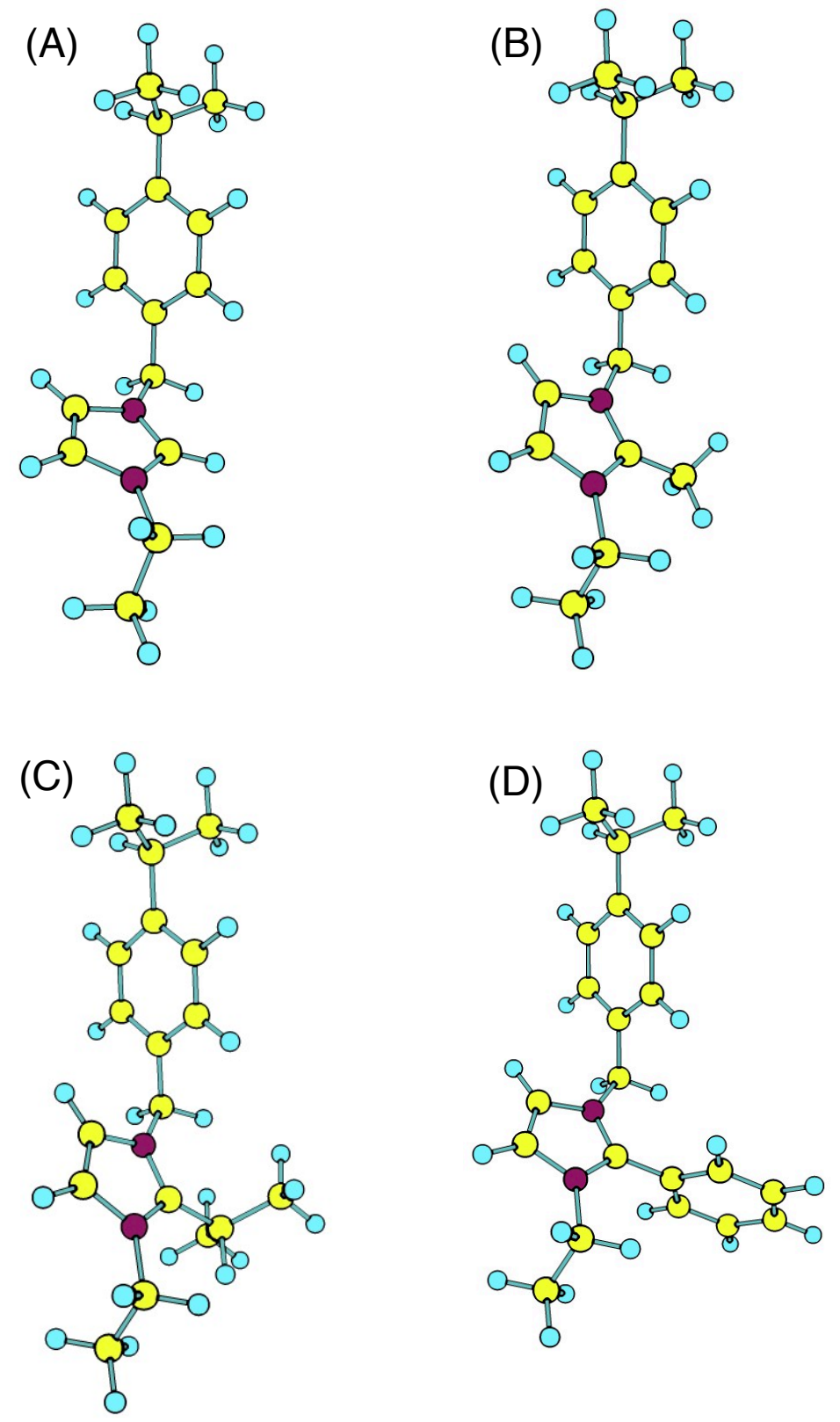

Figure 1: Calculated optimized geometries in imidazolium cations. (A) EIIM (C2unsubstituted). (B) EMIIM. (C) EIIIM. (D) EPhIIM. (B), (C) and (D) are substituted by methyl, isopropyl and phenyl groups. Yellow, blue and magenta spheres are $\mathrm{C}, \mathrm{H}$ and $\mathrm{N}$, respectively. 


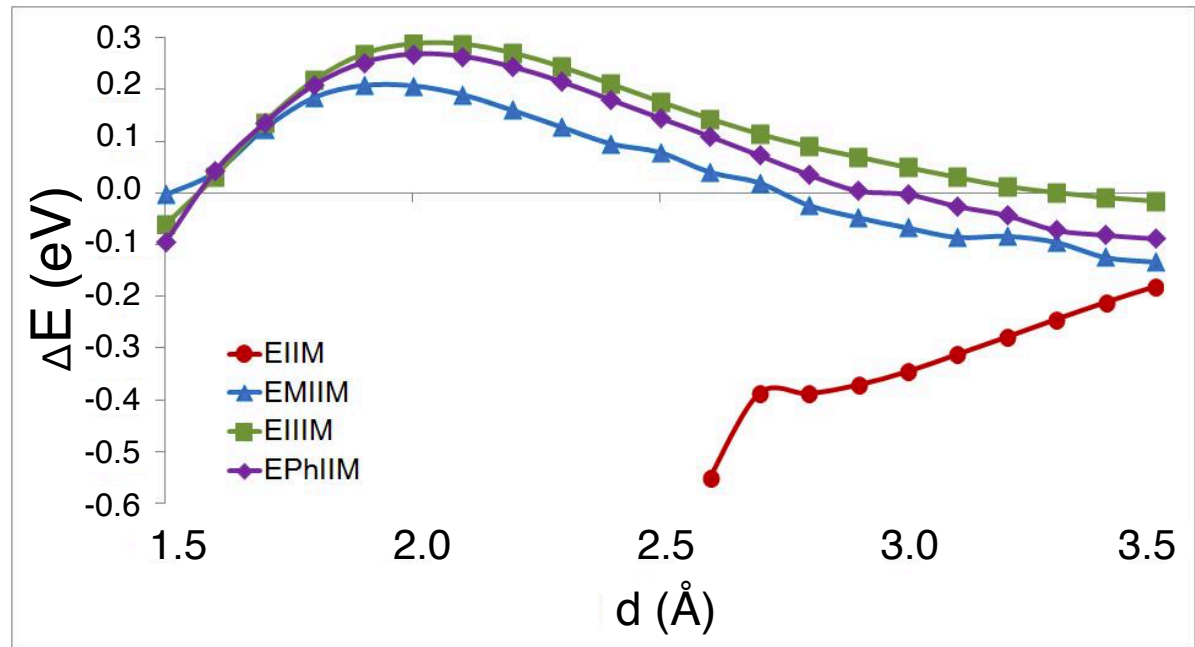

Figure 2: Calculated PECs. Effect on the steric hindrance appears except for EIIM. $d$ is fixed and changed from 3.5 to $1.5 \AA$ with every $0.1 \AA$. Energy is given by $\mathrm{eV}$. 


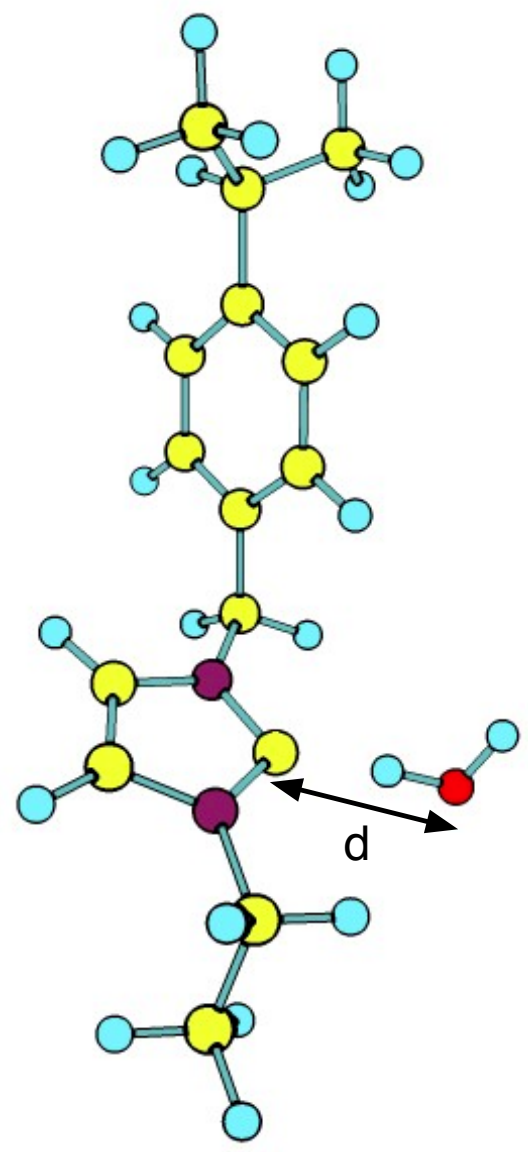

Figure 3: Calculated optimized geometry at $d=2.6 \AA$ in EIIM. EIIM is degraded at $d=2.6$ $\AA$ due to the deprotonation reaction. A red sphere is O. The black arrow shows $d$. 

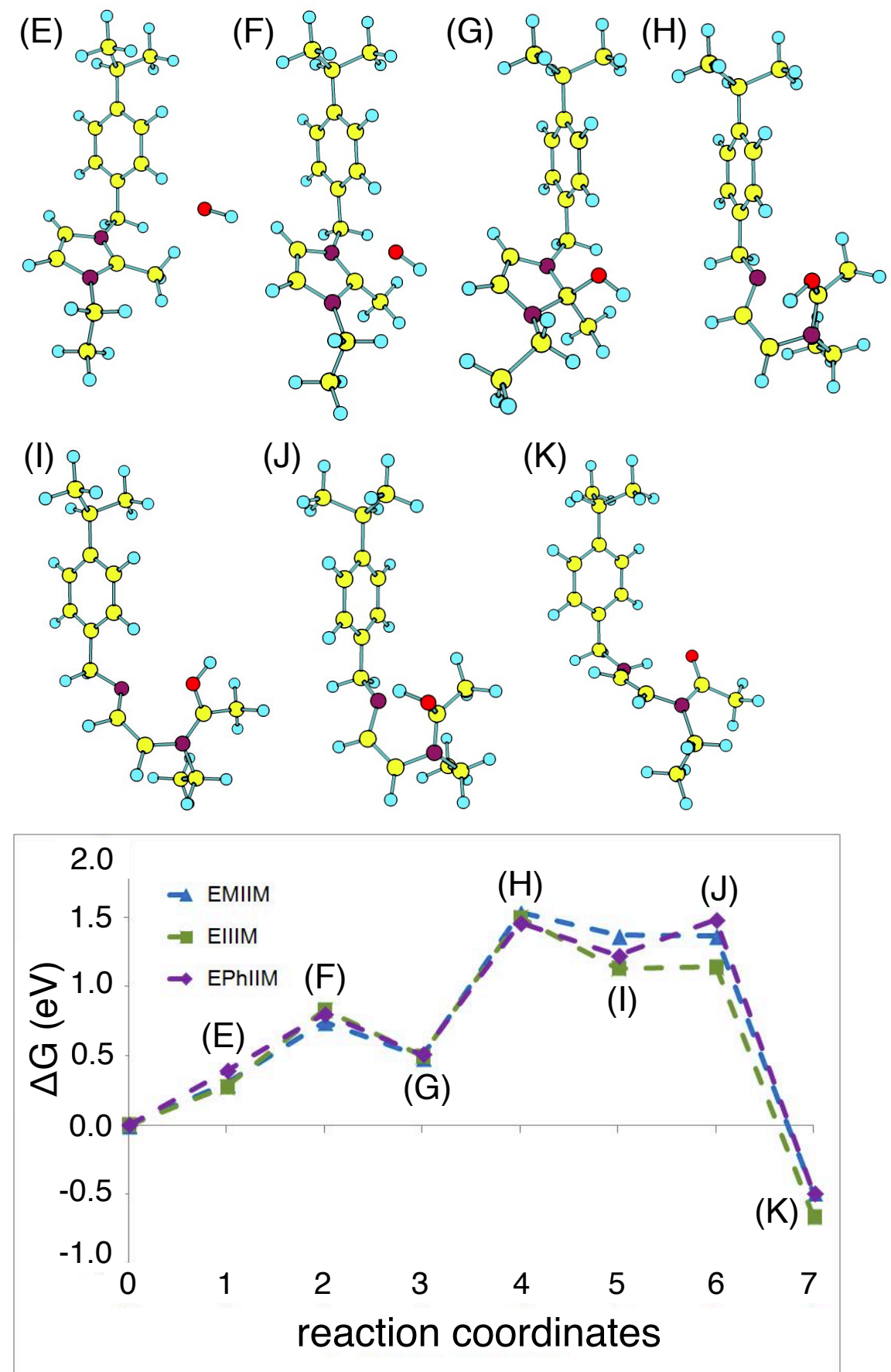

Figure 4: Calculated optimized geometries of EMIIM and minimum energy paths along the ring-opening reaction. (E) IS. (F) TS $\mathrm{TS}_{1}$ (G) intermediate state $\left(\mathrm{IMS}_{1}\right)$. (H) $\mathrm{TS}_{2}$. (I) $\mathrm{IMS}_{2}$. (J) $\mathrm{TS}_{3}$. (K) FS. Energy is given by eV. 


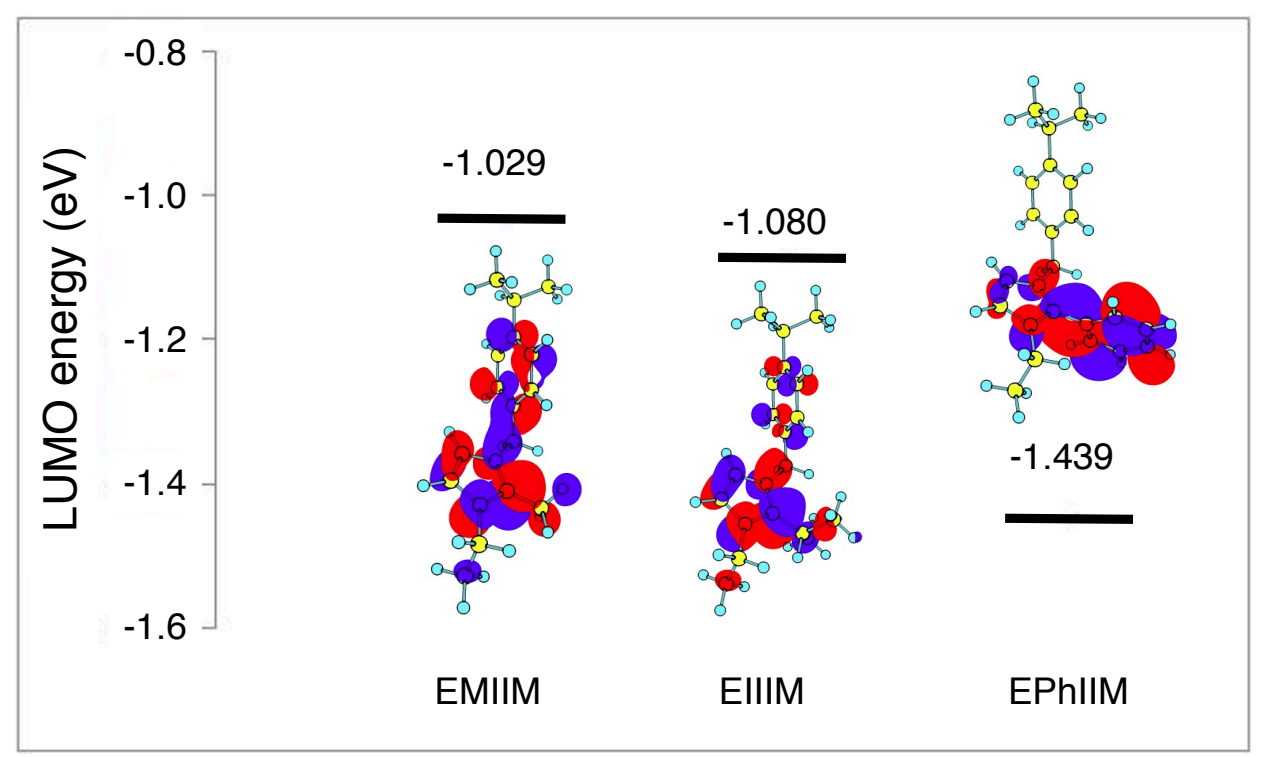

Figure 5: LUMO energy and isosurface of the C2-substituted imidazolium cations. The order of the LUMO energy corresponds to that of the energy barriers in the ring-opening reaction. Energy is given by eV. 


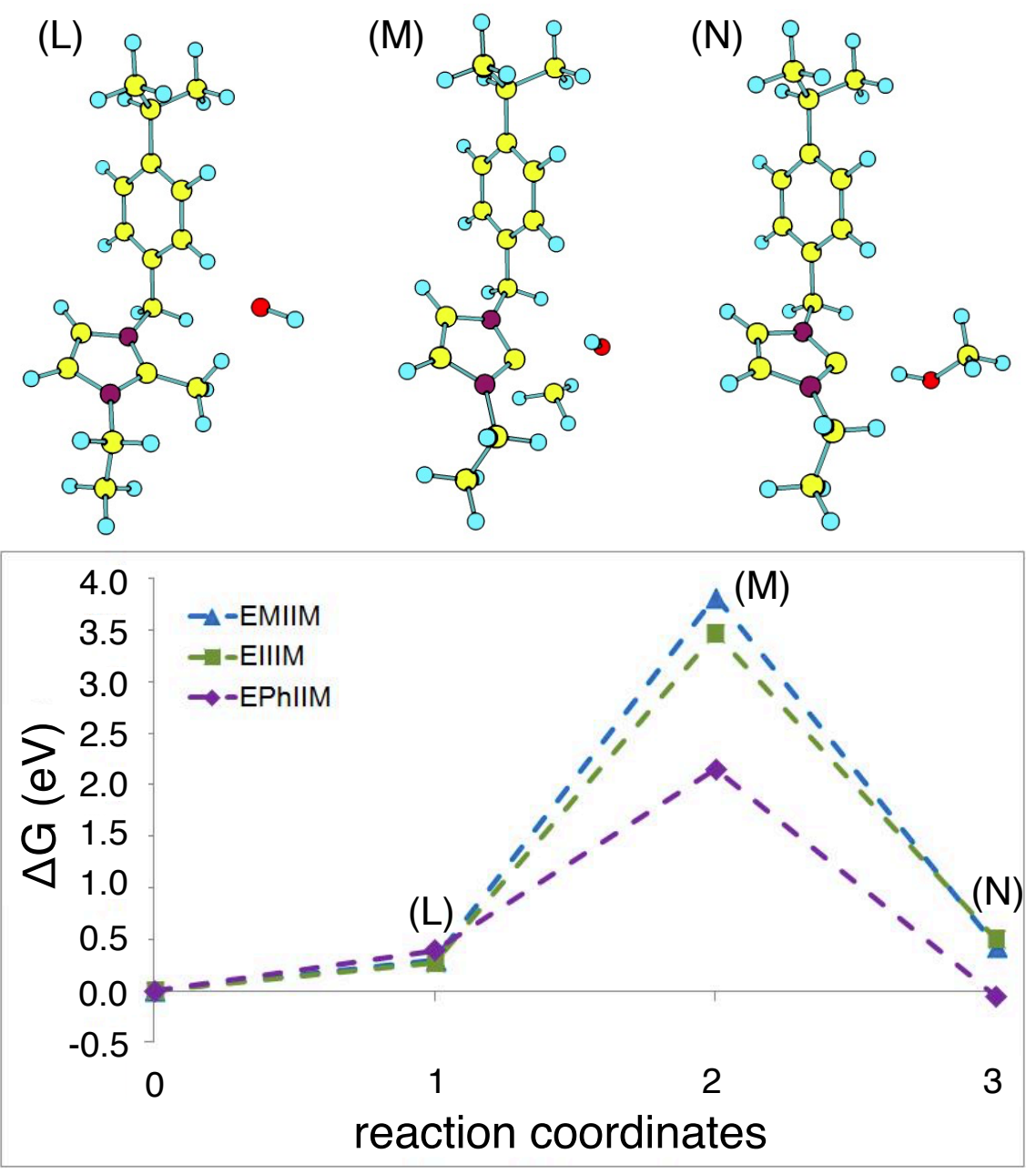

Figure 6: Calculated optimized geometries of EMIIM and minimum energy paths along the deprotonation reaction. (L) IS. (M) TS. (N) FS. Energy is given by eV. 